\title{
Editorial to the Topical Collection on Supernovae
}

\author{
A.M. Bykov ${ }^{1}$ - R.A. Chevalier ${ }^{2}$ J.C. Raymond ${ }^{3}$. \\ F.-K. Thielemann ${ }^{4,5} \cdot$ M. Falanga ${ }^{6} \cdot$ R. von Steiger ${ }^{6}$
}

Published online: 1 May 2018

(C) Springer Science+Business Media B.V., part of Springer Nature 2018

\section{Supernovae in Astrophysics}

Being one of the most luminous and spectacular phenomena in the sky, supernovae ( $\mathrm{SNe}$ ) have been actively studied since early Chinese astronomers left records of guest stars, to the most recent high precision, multi-wavelength observations. This intense study has yielded in recent years great insight into the physics of supernovae and their impact on a very wide variety of subjects from cosmology to astrobiology. The unprecedented growth of observational information, the ever increasing computational power for modeling, and the deep multi-disciplinary connections of supernovae to stellar physics, galactic evolution, astrochemistry, and cosmic ray origin has motivated the publication of this topical collection of reviews. Our aim is to provide some insight on current progress and present some open questions for future supernova studies.

We note that relativistic stellar mass objects, i.e., neutron stars and black holes, are the compact remnants of supernovae. Therefore, this "Supernovae" topical collection is generically connected to the topical collection "Jets and Winds in Pulsar Wind Nebulae, GammaRay Bursts and Blazars” published in Space Science Reviews, volume 207, 2017.

Supernovae

Edited by Andrei Bykov, Roger Chevalier, John Raymond, Friedrich-Karl Thielemann, Maurizio Falanga and Rudolf von Steiger

$\triangle$ A.M. Bykov

byk@astro.ioffe.ru

1 Ioffe Institute, 194021, St. Petersburg, Russia

2 Department of Astronomy, University of Virginia, P.O. Box 400325, Charlottesville, VA 22904-4325, USA

3 Center for Astrophysics, 60 Garden St., Cambridge, MA 02138, USA

4 Department of Physics, University of Basel, 4056 Basel, Switzerland

5 GSI Darmstadt, 64291 Darmstadt, Germany

6 International Space Science Institute, Bern, Switzerland 


\section{Supernovae in Cosmology}

Type Ia supernovae are used to accurately determine cosmological distances and this has helped determine the local expansion rate of the Universe leading to the very important discovery of the accelerated expansion of the Universe (Riess et al. 1998; Perlmutter et al. 1999). This has further led to the dark energy paradigm within general relativity which continues to challenge current concepts in fundamental physics. The assumptions that no selection biases affected the distance measurements, that the corrections applied to nearby Type Ia $\mathrm{SNe}$ are valid throughout the whole observed redshift range, and that the corrections for redshift and absorption can be controlled, are discussed in this collection of review papers by Leibundgut and Sullivan. These assumptions are critical for Type Ia cosmological applications. Leibundgut and Sullivan also discuss future studies with the ground-based Large Synoptic Survey Telescope (LSST) and the large space-based projects Euclid and WFIRST. The LSST should provide hundreds of thousands of transient candidates every night and likely find more than 100,000 Type Ia SNe in five years.

\section{Modeling of Core-Collapse and Thermonuclear Supernovae}

Apart from the classical problems of stellar evolution like nucleosynthesis, hydrodynamical transport and instabilities, radiative transfer and magnetic phenomena, the physics of supernovae also includes the accretion and jets of the compact objects with general relativistic effects and neutrinos and astroparticle phenomena. This makes the modeling of supernova explosions and evolution to be extremely complicated, but very exciting. Burrows et al present a review of crucial physical dependencies of the core-collapse supernova mechanism. They emphasize the role of the small changes in the neutrino-matter couplings in the proximity to criticality to produce sizable effects on the core-collapse. Nomoto and Leung review the single degenerate models for Type Ia supernovae. They discuss the evolution and nucleosynthesis yields of the white dwarfs in the binary star systems with non-degenerate companion stars. Röpke and Sim review the possible numerical approaches to model properly the multi-physics and the multi-scale nature of Type Ia supernovae and related astrophysical transients.

\section{Neutrinos in Supernovae}

Neutrinos play a fundamental role in the collapse of massive stars. Recent 2D and 3D simulations of core-collapse SNe have revealed that even modest changes in the neutrino-matter coupling may have profound effects on the resulting hydrodynamical matter motions associated with the energy release that may power the explosion. A large-scale asymmetric emission of the electron neutrino lepton number (LESA) discovered in 3D simulations may result in neutron-star kicks and affect the nucleosynthesis in the neutrino-driven winds. These processes, as well as the flavor conversions in the stellar envelopes, and the high energy neutrinos from the hidden relativistic outflows associated with the core collapse of massive stars, are reviewed by Tamborra and Murase. Since they are weakly interacting, neutrinos carry unique information on the accretion phase of the core collapse before the first photons can be detected from the supernova. This idea is realized in the SuperNova Early Warning System (SNEWS) project which involves an international network of supernova-neutrinosensitive detectors. Currently, seven neutrino experiments are involved in SNEWS: Super-K 
(Japan), LVD (Italy), Ice Cube (South Pole), KamLAND (Japan), Borexino (Italy), Daya Bay (China), and HALO (Canada). SNEWS will alert astronomers of the developing collapse. Detection of the neutrino signal, as well as the associated gravitational waves, will provide important new information to test the complex supernova models. Observational constraints on the neutrino flavor oscillations in the stellar envelope will test the neutrino oscillation physics.

\section{Superluminous and Unusual Supernovae}

Recent supernova searches have examined open areas of sky, as opposed to nearby galaxies as was done in earlier searches. The searches revealed rare, very luminous events, now called superluminous supernovae (SLSNe). Moriya, Sorokina, and Chevalier review the properties of these events and discuss the possible power sources for the emission, including radioactivity, circumstellar interaction, and magnetar power. Milisavljevic and Margutti discuss peculiar supernovae, starting with the observations that led to the recognition of Type $\mathrm{Ib} / \mathrm{c}$ supernovae. They go on to describe unusual events, including SN 2014C, which made a transition between supernova types. Analysis of optical observations of unusual events is often aided by multi-wavelength observations, especially at radio and X-ray wavelengths. This is also true for supernovae interacting with dense circumstellar media, which are discussed by P. Chandra. The dense media imply a high rate of mass loss just before the supernova that is not understood in standard stellar evolution theory.

\section{Supernova Remnants}

Higher and higher spatial and spectral resolution observations of supernova remnants are becoming available at gamma-ray through radio wavelengths. Lopez and Fesen review how the morphology and kinematics of SNRs can be used to determine the supernova type and to explore the nature of the explosion through signatures such as jets and bubbles in the ejecta. The shock waves that make the gas visible are different from shocks in gas of normal astrophysical abundance due to the enormous radiative cooling rates in gas devoid of $\mathrm{H}$ and $\mathrm{He}$, and Raymond reviews the physical processes and their consequences for observable spectra.

\section{Nucleosynthesis and Dust Formation in Supernovae}

While the general picture of the nucleosynthesis in supernovae has been understood for quite a while, some major open questions remains. These are related to the precise amounts of alpha-elements produced in core-collapse $\mathrm{SNe}$, the detailed composition of the $\mathrm{Fe}$-group and the respective role of Type Ia vs. the core-collapse of massive stars. Thielemann, Isern, Perego and von Ballmoos present a detailed review of the status of nucleosynthesis in both Type Ia and core-collapse supernovae and the open problems responsible for the production of the intermediate mass, Fe-group and heavier elements. They discussed the wide range of the observational constraints on the nucleosynthesis in supernovae.

Dust can be produced efficiently in supernovae and supernova remnants. The dust formation scenarios are reviewed in detail by Sarangi, Matsuura and Micelotta. A review of the state of the art results of the dust processing and survival inside supernova remnants, in terms of theoretical modelling and with comparison to observations, is presented by Micelotta et al. 


\section{Cosmic Rays in Supernovae}

The early suggestion by Baade and Zwicky (1934) that cosmic rays (CRs) are produced by supernovae has now a conclusive confirmation in radio, X-ray, and gamma-ray observations of individual supernova remnants (SNRs). It has become clear that SNe are the most likely source of cosmic rays with energies below the so-called "knee" at $\sim 10^{15} \mathrm{eV}$. The origin of CRs with energies beyond the knee is less certain. Bykov, Ellison, Marcowith and Osipov review the possible mechanisms of $\mathrm{CR}$ acceleration both below the knee and well beyond $\mathrm{PeV}$ energies. The observed energy density and composition of $\mathrm{GeV}$ cosmic rays imply that the mechanism of cosmic ray acceleration must be highly efficient with at least $10 \%$ of the total kinetic energy of the supernova ejecta going into CRs if they are powered by supernova shocks. If the acceleration efficiency is this high, feedback effects from CR production will influence the shock dynamics. Furthermore, since CRs are connected to the supernova outflows through fluctuating magnetic fields, a high acceleration efficiency is likely to lead to strong magnetic field amplification in fast supernova shocks. Models of Fermi type acceleration in fast supernova shocks with magnetic field amplification, including trans-relativistic $\mathrm{SNe}$, are reviewed.

The circumstellar environment can play a role in high-energy CR acceleration. The role of superluminous interaction powered $\mathrm{SNe}$, and normal core-collapsed $\mathrm{SNe}$ in clusters of young massive stars, in efficiently producing CRs to PeV energies is discussed. It is further suggested that galactic sources associated with infrequent but powerful relativistic $\mathrm{SNe}$ can accelerate $\mathrm{CR}$ protons up to $\mathrm{EeV}$ energies (i.e., $10^{18} \mathrm{eV}$ ).

Overall, this collection of papers provides a timely and comprehensive review of the multi-wavelength observations and the main physical processes involved in the study of supernovae and supernova remnants with applications to nucleosynthesis and cosmic ray physics. It will be particularly useful to postgraduate students and researchers active in various areas of astrophysics and space science. This collection is based on an international workshop which took place at ISSI Bern during October 3-7 in 2016 where about forty leading experts in physics and astrophysics of supernovae and supernova remnants discussed the issues presented here. 